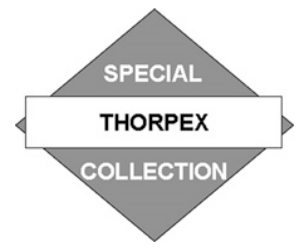

\title{
Predictability of an Atmospheric Blocking Event that Occurred on 15 December 2005
}

\author{
Mio MATSUEDA \\ Japan Agency for Marine-Earth Science and Technology, Tsukuba, Japan \\ MASAYUKI KYOUDA \\ Numerical Prediction Division, Japan Meteorological Agency, Tokyo, Japan \\ ZOLTAN TOTH \\ NOAA/Earth Systems Research Laboratory/Global Systems Division, Boulder, Colorado \\ H. L. TANAKA \\ Center for Computational Sciences, University of Tsukuba, Tsukuba, Japan \\ TADASHI TSUYUKI \\ Meteorological Research Institute, Tsukuba, Japan
}

(Manuscript received 1 July 2010, in final form 18 November 2010)

\begin{abstract}
Atmospheric blocking occurred over the Rocky Mountains at 1200 UTC 15 December 2005. The operational medium-range ensemble forecasts of the Canadian Meteorological Center (CMC), the Japan Meteorological Agency (JMA), and the National Centers for Environmental Prediction (NCEP), as initialized at 1200 UTC 10 December 2005, showed remarkable differences regarding this event. All of the NCEP members failed to predict the correct location of the blocking, whereas almost all of the JMA members and most of the CMC members were successful in predicting the correct location. The present study investigated the factors that caused NCEP to incorrectly predict the blocking location, based on an ensemble-based sensitivity analysis and the JMA global spectral model (GSM) multianalysis ensemble forecasts with NCEP, regionally amplified NCEP, and globally amplified NCEP analyses.

A sensitive area for the blocking formation was detected over the central North Pacific. In this area, the NCEP control analysis experienced problems in the handling of a cutoff cyclone, and the NCEP initial perturbations were ineffective in reducing uncertainties in the NCEP control analysis. The JMA GSM multianalysis ensemble forecasts revealed that regional amplification of initial perturbations over the sensitive area could lead to further improvements in forecasts over the blocking region without degradation of forecasts over the Northern Hemisphere (NH), whereas the global amplification of initial perturbations could lead to improved forecasts over the blocking region and degraded forecasts over the NH. This finding may suggest that excessive amplification of initial perturbations over nonsensitive areas is undesirable, and that case-dependent rescaling of initial perturbations may be of value compared with climatology-based rescaling, which is widely used in current operational ensemble prediction systems.
\end{abstract}

Corresponding author address: Mio Matsueda, Japan Agency for Marine-Earth Science and Technology, 1-1 Nagamine, Tsukuba, Ibaraki 305-0052, Japan.

E-mail: mimatsue@mri-jma.go.jp

\section{Introduction}

Atmospheric blocking is an important weather regime in midlatitude weather and climate, as persistent blocking can induce extremely high or low temperatures and 
severe precipitation anomalies over the surrounding area. Namias (1947) first attempted to identify the synoptic characteristics of blocking in an analysis of the anomalous winter of 1946/47, and Rex (1950) first proposed a set of criteria for identifying blocking events. Subsequently, many studies have investigated extreme weather events related to blocking, the mechanism of blocking, and model performance in simulating blocking (Matsueda 2011, 2009; Matsueda et al. 2010, 2009; Tyrlis and Hoskins 2008; Black et al. 2004; Carrera et al. 2004; Mauritsen and Källén 2004; Trigo et al. 2004; Pelly and Hoskins 2003a,b; Quadrelli et al. 2001; Cash and Lee 2000; D'Andrea et al. 1998; Nakamura et al. 1997; Kimoto et al. 1992; Tanaka and Milkovich 1990; Shutts 1986, 1983).

It is well known that general circulation models tend to underestimate blocking frequency in numerical weather prediction (NWP) and climate projection (Palmer et al. 2008; Mauritsen and Källén 2004; Pelly and Hoskins 2003b; D'Andrea et al. 1998). The accurate simulation and prediction of atmospheric blocking has remained an open question in NWP and climate projection, as is the case for tropical and extratropical cyclones. In this regard, a research priority is investigations of blocking predictability and improvements in blocking prediction in mediumrange forecasts, for which the initial-value problem is of greater concern than the boundary-value problem. Advances in these areas may lead to improvements not only in medium-range forecasting skill but also in model performance in climate projections.

The NWP technique has progressed rapidly with advances in computer science. A 5-day weather forecast today is as reliable as a 2-day weather forecast 20 years ago, which represents a major scientific advance [(World Meteorological Organization) WMO 2006]. In recent years, ensemble forecasts have become a major component of operational global weather-prediction systems, gaining increasing attention at various time scales (short, medium, and long range) for both operational and research purposes.

In ensemble forecasting, multiple forecasts are performed by introducing perturbations in the initial conditions, in the boundary conditions or in the models themselves, mainly in order to estimate of the reliability of the forecast, which, because of changes in atmospheric predictability, varies from day to day and from region to region (Kalnay 2003).

The WMO began The Observing System Research and Predictability Experiment (THORPEX; WMO 2005) project in 2005 to accelerate improvements in the accuracy of 1-day to 2-week forecasts of high-impact weather for the benefit of society, the economy, and the environment. At the heart of THORPEX is the research needed for the design and demonstration of a Global Interactive Forecasting System (GIFS) that allows information exchange among the forecast users, numerical forecast models, data assimilation systems, and observations. The objective of the future GIFS is the production of internationally coordinated advance warnings and forecasts for high-impact weather events to mitigate loss of life and property, and to contribute to the welfare of all WMO nations (more information available online at http://tigge.ecmwf.int/research/gifs.html). GIFS is planned to be conducted operationally. The THORPEX Interactive Grand Global Ensemble (TIGGE; Richardson et al. 2005) is a key component of THORPEX, providing operational medium-range ensemble forecast data at close to real time (maximum ensemble size of 557). The key objectives of TIGGE are briefly as follows:

- An enhanced collaboration on development of ensemble prediction, internationally and between operational centers and universities;

- A deeper understanding of the contribution of observation, initial, and model uncertainties to forecast error;

- Test concepts of a TIGGE Prediction Centre to produce ensemble-based predictions of high-impact weather, wherever it occurs, on all predictable time ranges;

- The development of a prototype future GIFS.

As of July 2010, 10 operational NWP centers [the Bureau of Meteorology (BoM; Australia), the China Meteorological Administration (CMA), the Canadian Meteorological Center (CMC), Centro de Previsão de Tempo e Estudos Climáticos (CPTEC; Brazil), the European Centre for Medium-Range Weather Forecasts (ECMWF), the Japan Meteorological Agency (JMA), the Korea Meteorological Administration (KMA), Météo-France, the National Centers for Environmental Prediction (NCEP), and the Met Office (UKMO)] were producing daily global ensemble forecasts (1-2 weeks ahead), delivering in near-real time a selection of forecast data to the TIGGE portals at CMA, ECMWF, and the National Center for Atmospheric Research (NCAR). More than 3 years have passed since the TIGGE portals came into operation in October 2006; however, few studies have made use of the operational medium-range ensemble forecast data provided by TIGGE portals (e.g., Froude 2010; Majumdar and Finocchio 2010; Matsueda 2011, 2009; Johnson and Swinbank 2009; Matsueda and Tanaka 2008; Pappenberger et al. 2008; Park et al. 2008).

Prior to the TIGGE project, Matsueda et al. (2006, 2007) constructed the Multicenter Grand Ensemble (MCGE), consisting of three operational medium-range ensemble forecasts (maximum ensemble size of 86) by CMC, JMA, and NCEP, on a quasi-operational basis. They demonstrated the advantage of MCGE over a 
TABLE 1. Operational medium-range ensemble prediction systems at CMC, JMA, and NCEP as of December 2005.

\begin{tabular}{lccc}
\hline \hline & CMC Canada & JMA Japan & NCEP United States \\
\hline Model uncertainty & Multimodel, stochastic physics & None & None \\
Initial perturbation & EnKF & BVs & BVs \\
Forecast model resolution & TL149L23-41, $1.2^{\circ}$ L28 & T106L40 & T126L28 \\
Initial time & $0000 \mathrm{UTC}$ & $1200 \mathrm{UTC}$ & $0000,0600,1200,1800 \mathrm{UTC}$ \\
Forecast length & $240 \mathrm{~h}$ & $216 \mathrm{~h}$ & $384 \mathrm{~h}$ \\
Members per run & 17 & 25 & 11 \\
Members per day & 17 & 25 & 44 \\
\hline
\end{tabular}

single-center ensemble using monthly and daily deterministic and probabilistic scores for $500-\mathrm{hPa}$ geopotential height (Z500) and 850-hPa temperature over the Northern Hemisphere $\left(\mathrm{NH} ; 20^{\circ}-90^{\circ} \mathrm{N}\right)$. Matsueda et al. (2007) also showed a remarkable example of a medium-range ensemble forecast of atmospheric blocking that occurred over the Rocky Mountains on 15 December 2005. All the NCEP members failed to predict the correct location of blocking, whereas almost all the JMA members and most of the CMC members predicted the correct location. The factors that underlie the collective failure of NCEP members to predict the blocking location remain to be determined. The aim of this study is to identify these factors using an ensemble-based simple sensitivity analysis and JMA global spectral model (GSM) multianalysis ensemble forecasts.

The remainder of this manuscript is organized as follows. Section 2 introduces the three operational medium-range Ensemble Prediction Systems (EPSs) as of December 2005 , and outlines the analysis procedures employed in this study. Section 3 presents the results of analyses of atmospheric blocking using ensemble forecast data, an ensemble-based simple sensitivity analysis, and JMA GSM multianalysis ensemble forecasts. Finally, the conclusions are presented in section 4 .

\section{Data and method}

\section{a. Ensemble forecast data}

Three sets of operational medium-range ensemble forecast data are employed: CMC, JMA, and NCEP. The details of these EPSs, as of December 2005, are summarized in Table 1. Most of the EPS configurations shown in Table 1 are different from those currently employed (for the latest information see http://tparc.mri-jma. go.jp/TIGGE/data_details.pdf) because of the remarkable recent development of ensemble forecast and computer technology. Note that the following descriptions of EPSs relate to EPSs as of December 2005 rather than current versions.

The CMC EPS was initialized at 0000 UTC with 17 ensemble members and a multimodel approach. CMC added random perturbations to the observations and generated perturbed analyses using an ensemble Kalman filter (EnKF; Houtekamer and Mitchell 2005). The CMC EPS was conducted using eight different versions of the spectral finite-element model (SEF; Ritchie 1991; Ritchie and Beaudoin 1994) and eight different versions of the Global Environmental Multiscale model (GEM; Côté et al. 1998a,b). The models differ in their physical parameterizations and their dynamical cores. The horizontal resolutions of the SEF and GEM models are TL149 and $1.2^{\circ}$, respectively, being largely equivalent. The JMA EPS was initialized at 1200 UTC with 25 ensemble members (JMA 2007), using bred vectors (BVs) as initial perturbations. A low-resolution version of the JMA GSM, T106L40, was integrated from 1 unperturbed and 24 perturbed initial conditions. Thus, the dynamical framework and physical processes were identical with those of the operational GSM except for the horizontal resolution. NCEP and ECMWF first started mediumrange ensemble forecasts in December 1992. NCEP originally developed the BV method for medium-range ensemble forecasts (Toth and Kalnay 1993, 1997). The NCEP EPS was conducted with 11 ensemble members based on BVs, 4 times daily $(0000,0600,1200$, and 1800 UTC). The horizontal resolution and vertical levels of the model are TL126L28.

\section{b. Ensemble-based sensitivity analysis}

Enomoto et al. (2007) proposed a simple singularvector-like sensitivity analysis using only existing ensemble forecast data. This method does not require a numerical prediction model, tangent-linear model, or adjoint code, and requires only ensemble forecast data calculated in advance. This technique can be used to identify a sensitivity area that is expected to affect the prediction of a particular atmospheric phenomenon over a target area. A brief description of the method is as follows.

The goal of this technique is to find the fastest-growing perturbation $\mathbf{x}$. The time evolution of $\mathbf{x}$ is represented as

$$
\mathbf{z}=M \mathbf{x},
$$


where $\mathbf{z}$ is a forecast departure from the control run at a target lead time for the perturbation $\mathbf{x}$, and $M$ is a numerical model. The time evolution for each ensemble member is assumed as follows:

$$
\mathbf{z}_{i}=M \mathbf{y}_{i}, \quad i=1,2, \ldots, n,
$$

where $\mathbf{z}_{i}$ is the forecast departure from the control run at a target lead time for the $i$ th member, and $\mathbf{y}_{i}$ is the initial perturbation for the $i$ th member. Consider a linear combination of the original initial perturbations to find the fastest-growing initial perturbation $\mathbf{x}$ :

$$
\mathbf{x}=p_{1} \mathbf{y}_{1}+p_{2} \mathbf{y}_{2}+\cdots+p_{n} \mathbf{y}_{n}
$$

Using matrix notation:

$$
\begin{aligned}
& \mathbf{Y}=\left(\mathbf{y}_{1} \mathbf{y}_{2} \ldots \mathbf{y}_{n}\right), \\
& \mathbf{Z}=\left(\mathbf{z}_{1} \mathbf{z}_{2} \ldots \mathbf{z}_{n}\right), \\
& \mathbf{p}=\left(p_{1} p_{2} \ldots p_{n}\right)^{\mathrm{T}},
\end{aligned}
$$

Eqs. (2) and (3) may be written as

$$
\begin{aligned}
& \mathbf{Z}=M \mathbf{Y}, \\
& \mathbf{x}=\mathbf{Y} \mathbf{p},
\end{aligned}
$$

and Eq. (1) can then be written as

$$
\mathbf{z}=M \mathbf{x}=M \mathbf{Y p}=\mathbf{Z p} .
$$

Note that a numerical model $M$ was replaced with the forecast departure $\mathbf{Z}$. The constrained maximization problems for the norm of $\mathbf{z},\|\mathbf{z}\|$, can be solved using Lagrange multipliers. The Lagrange function and its variations are represented as follows:

$$
\begin{aligned}
F(\mathbf{p}, \lambda) & =\langle\mathbf{z}, \mathbf{z}\rangle+\lambda(1-\langle\mathbf{x}, \mathbf{x}\rangle) \\
& =\langle\mathbf{Z} \mathbf{p}, \mathbf{Z} \mathbf{p}\rangle+\lambda(1-\langle\mathbf{Y} \mathbf{p}, \mathbf{Y} \mathbf{p}\rangle) \\
\delta F(\mathbf{p}, \lambda) & =2\left\langle\delta \mathbf{p}, \mathbf{Z}^{\mathrm{T}} \mathbf{Z} \mathbf{p}-\lambda \mathbf{Y}^{\mathrm{T}} \mathbf{Y} \mathbf{p}\right\rangle-\delta \lambda(1-\langle\mathbf{Y} \mathbf{p}, \mathbf{Y} \mathbf{p}\rangle)
\end{aligned}
$$

As a result, it is only necessary to solve the following eigenvalue problem:

$$
\left(\mathbf{Y}^{\mathrm{T}} \mathbf{Y}\right)^{-1} \mathbf{Z}^{\mathrm{T}} \mathbf{Z} \mathbf{p}=\lambda \mathbf{p}
$$

The size of the matrix $\left(\mathbf{Y}^{\mathrm{T}} \mathbf{Y}\right)^{-1} \mathbf{Z}^{\mathrm{T}} \mathbf{Z}$ is equal to the ensemble size $[\sim O(10)]$. The eigenvalue problem is easily solved. If each initial perturbation is mutually orthogonal and has the same norm, $\left(\mathbf{Y}^{\mathrm{T}} \mathbf{Y}\right)^{-1}$ becomes a scalar matrix. It is only necessary to perform the singular value decomposition of the matrix $\mathbf{Z}$. Note that it is only necessary to use half of the original initial perturbations if an NWP center uses positive-negative perturbation pairs.

\section{c. JMA GSM multianalysis ensemble forecasts}

Based on the results of ensemble-based simple sensitivity analysis, multianalysis ensemble forecasts were performed using the JMA GSM (JMA 2007). The JMA GSM used here is the same as the operational GSM used in the JMA EPS during the period March 2006November 2007. The horizontal resolution and vertical levels of the JMA GSM are TL159L40. In multianalysis ensemble forecasts, three kinds of analyses were used: NCEP control and perturbed analyses; globally amplified NCEP analyses, which consist of the NCEP control analysis and globally amplified (by a factor of 1.5) NCEP initial perturbations; and regionally amplified NCEP analyses with regionally amplified (by a factor of 1.5 ) NCEP initial perturbations, although only over a sensitive area detected by the ensemble-based sensitivity analysis.

\section{Results}

\section{a. Blocking that occurred at 1200 UTC 15 December 2005}

Blocking started to develop over the Rocky Mountains on 13 December 2005 and started to have a remarkable meandering shape at 1200 UTC 15 December 2005. Blocking reached full maturity on 18 December 2005, but decayed within several days. According to the Tibaldi and Molteni (TM) blocking index (Tibaldi and Molteni 1990) that is widely used in many studies, a blocking event was detected during 13-21 December 2005. The definition of the TM index is essentially derived from the work of Lejenäs and Økland (1983). The 500-mb fields during 13-21 December 2005 also satisfied the criteria of normalized 500-mb anomaly shown in Dole (1986), although the duration is shorter than 10 days. [The minimum duration for blocking definition is different among studies (mostly 5 to 10 days).] The operational medium-range ensemble forecasts of CMC, JMA, and NCEP, valid at 1200 UTC 15 December 2005, initialized on 10 December 2005, showed remarkable differences regarding this event.

Figure 1 shows spaghetti diagrams at Z500 for the CMC, JMA, and NCEP ensemble forecasts and JMA analysis, valid at 1200 UTC 10-15 December 2005. The initial times are 1200 UTC 10 December 2005 for the JMA and NCEP forecasts, and 0000 UTC 10 December 2005 for the CMC forecast. Note that forecast times used 
(a) CMC

(b) JMA

(c) NCEP

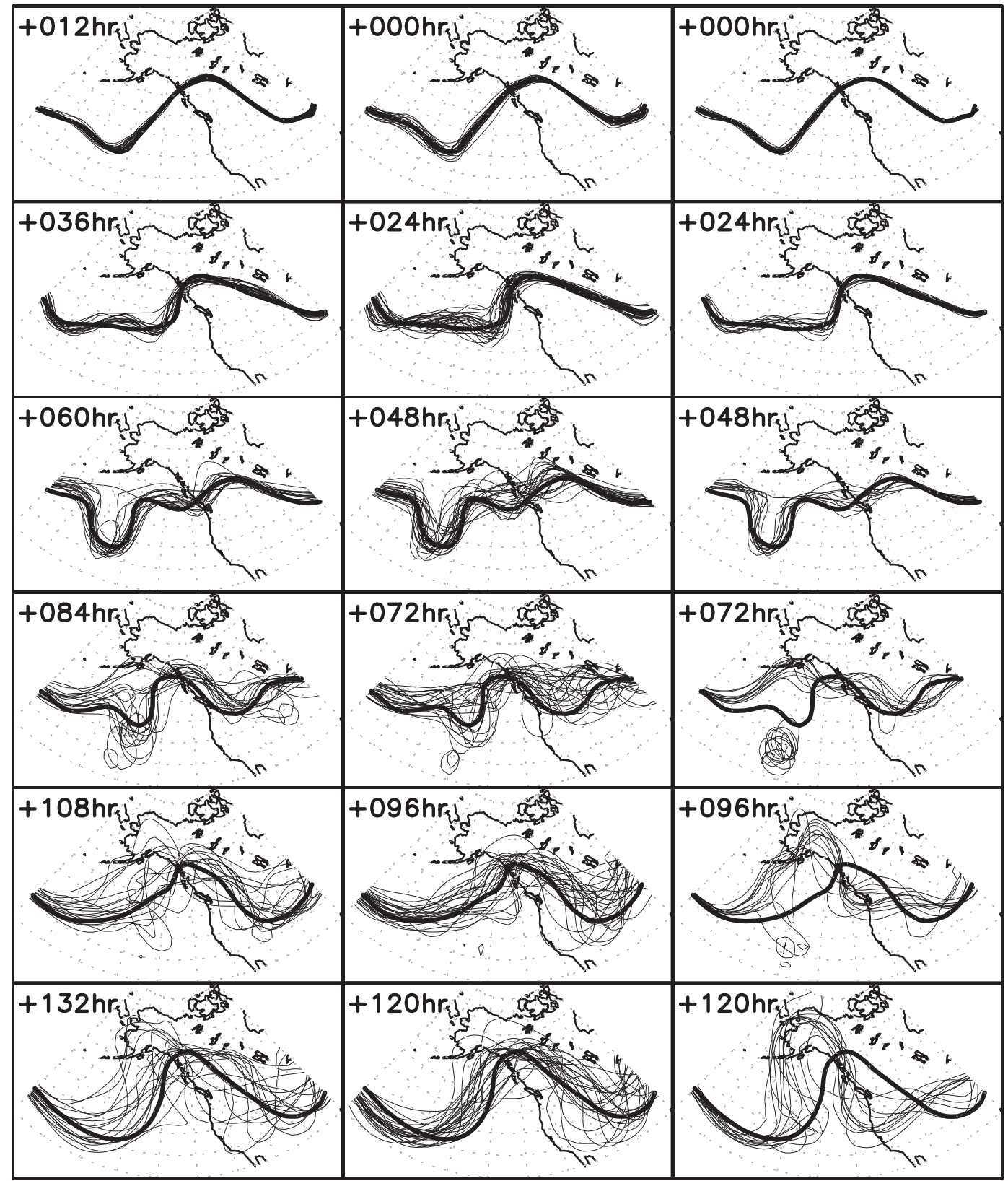

FIG. 1. Spaghetti diagrams for 500-hPa height $(5500 \mathrm{~m}$ ) for ensemble members (thin line) of (a) CMC, (b) JMA, and (c) NCEP, initialized at 0000 (CMC) or 1200 UTC (JMA and NCEP) 10 Dec 2005. Forecast times are shown in the upper left of (a)-(c). The thick lines are for the JMA analysis.

hereafter are for JMA and NCEP. Up to a lead time of $48 \mathrm{~h}$, all ensemble members perform well in capturing the analysis. However, for a 72-h lead time, all the NCEP members and some of the CMC members start to predict a pseudoblocking over the area south of Alaska. For a 96-h lead time, these members predicted a ridge of blocking located upstream of the analysis. For a $120-\mathrm{h}$ lead time, the members predicted a blocking event over Alaska, whereas almost all of the JMA members and most of the CMC members correctly predicted a blocking event over the Rocky Mountains.

In terms of the ensemble forecasts initialized on 11 December 2005, most of the CMC members and half of the JMA members incorrectly predicted the location 


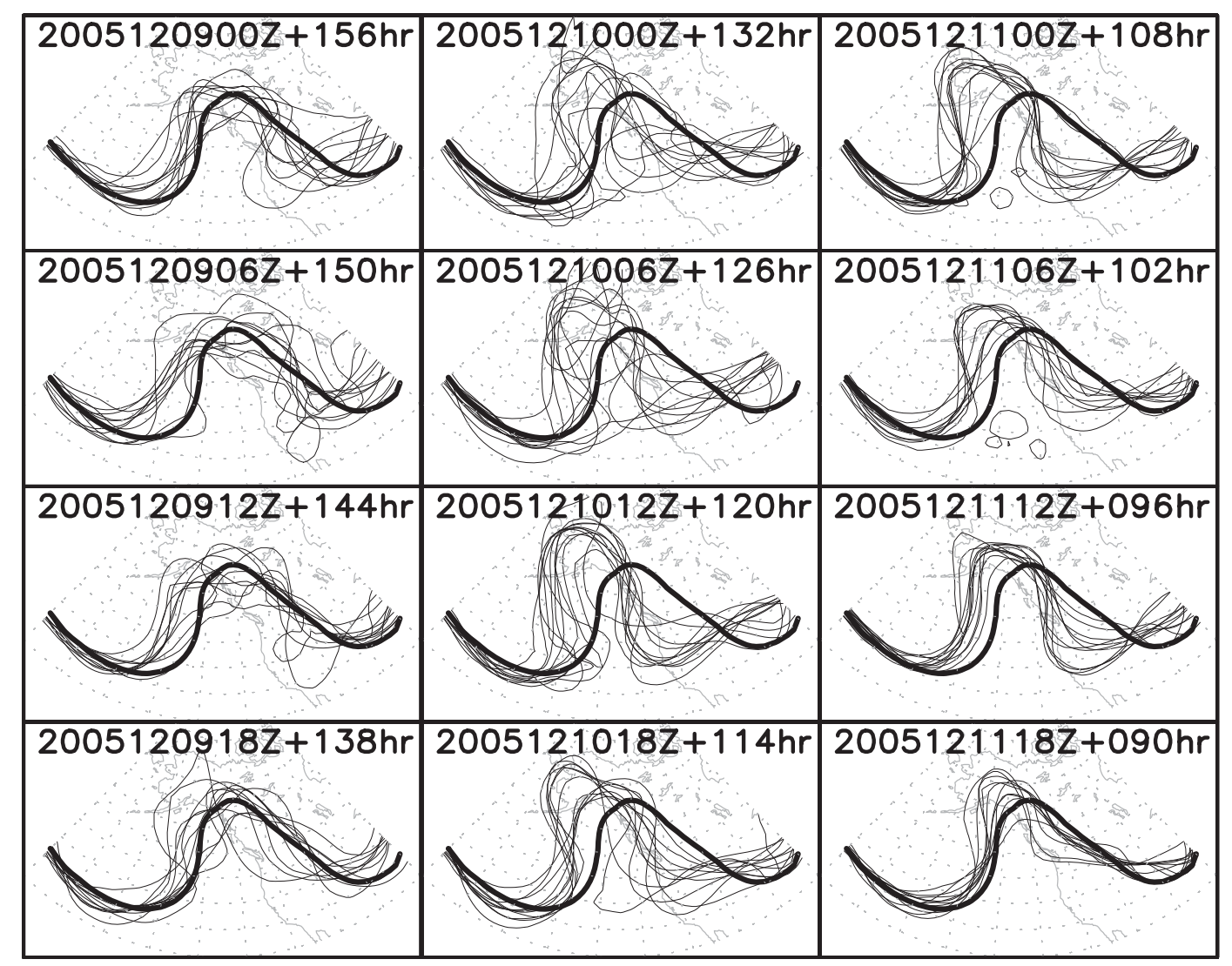

FIG. 2. Spaghetti diagrams for 500-hPa height $(5500 \mathrm{~m})$ for the NCEP ensemble members, valid at 1200 UTC 15 Dec 2005, initialized on (left) 9 Dec 2005, (middle) 10 Dec 2005, and (right) 11 Dec 2005. The thick lines show the NCEP analysis.

of the blocking (data not shown), as was the case for NCEP members initialized at 1200 UTC 10 December 2005. In addition, the NCEP members initialized during the period 9-11 December 2005 showed remarkable differences (Fig. 2). Most of the members initialized on 9 December predicted the correct location of blocking. In contrast, the members initialized on 10 December 2005 performed relatively poorly in this regard. For members initialized after 1800 UTC 10 December 2005, the predicted blocking locations showed a gradual improvement with later initial time of the ensemble forecast.

Because of limitations in the data, only the JMA and NCEP ensemble forecasts initialized at 1200 UTC 10 December 2005 are used hereafter.

\section{b. Comparison of control runs}

Given that almost all the JMA members predicted the blocking location correctly and all the NCEP members predicted it incorrectly, it would be meaningful to focus on each unperturbed forecast (control run). Figure 3 shows the time evolution of (Fig. 3a) the JMA analysis, (Fig. 3b) the JMA control run, (Fig. 3c) the NCEP control run, and (Fig. 3d) the JMA GSM run with NCEP control analysis, valid at 1200 UTC 10-15 December 2005. The root-mean-square error (RMSE) shown in each panel was calculated using each analysis, which is defined as each control run at the initial time, over the blocking region $\left(20^{\circ}-80^{\circ} \mathrm{N}, 170^{\circ} \mathrm{E}-100^{\circ} \mathrm{W}\right)$.

The JMA control run (Fig. 3b) correctly predicted the blocking location (although of course, the JMA control run was not a perfect forecast). The NCEP control run (Fig. 3c) shows a similar time evolution to that of the JMA control run (Fig. 3b) until a lead time of 48 h. The RMSEs are similar between the control runs. For lead times greater than $48 \mathrm{~h}$, however, the evolution of the NCEP control run is different from that of the JMA control run. The NCEP control run generated a cutoff cyclone with positive forecast errors to the north and negative forecast errors to the south over the North Pacific for lead times of $48-72 \mathrm{~h}$. The blocking ridge in the NCEP control run started to develop in an area located upstream of the analyzed ridge. In contrast, the JMA control run did not produce a cutoff cyclone or the related positive and negative forecast errors over 


\section{(a) JMA-EPS analysis}

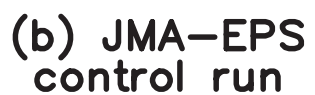

(c) NCEP-EPS control run

\section{(d) JMA-GSM run from NCEP cntl.}
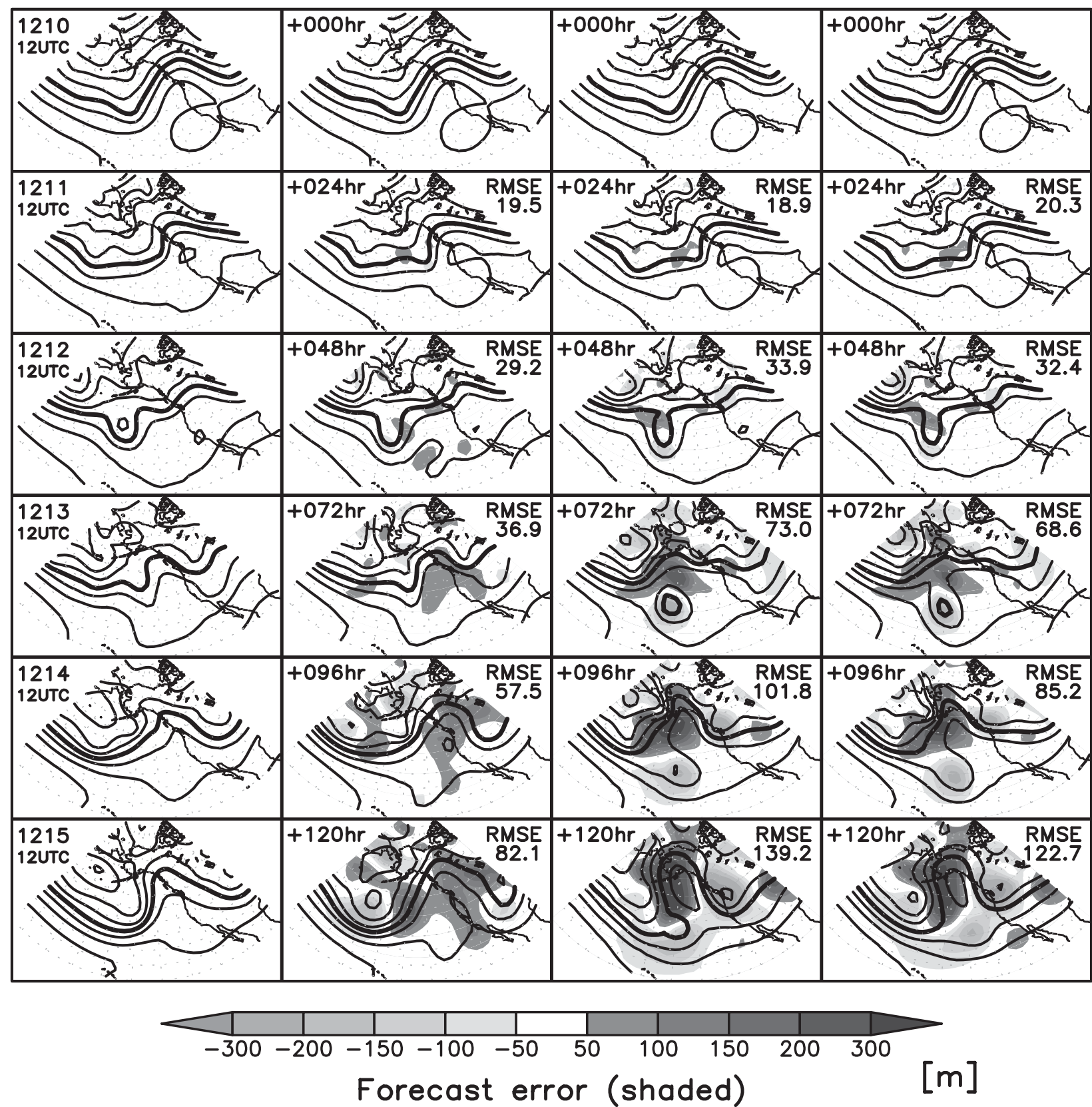

FIG. 3. Time evolution of 500-hPa height (contours) and forecast errors (shading: forecast minus analysis) for (a) JMA analysis, (b) JMA EPS control run, (c) NCEP EPS control run, and (d) JMA GSM run from the NCEP control analysis. The initial time is 1200 UTC 10 Dec 2005. The thick contours indicate $5500 \mathrm{gpm}$. RMSE [unit: geopotential meter (gpm)] is calculated using each analysis, which is defined as each control run at the initial time, over the blocking region $\left(20^{\circ}-80^{\circ} \mathrm{N}, 170^{\circ} \mathrm{E}-100^{\circ} \mathrm{W}\right)$.

the North Pacific, possibly explaining the correct development of blocking over the Rocky Mountains. The RMSE for the NCEP control run was about twice as large as that for the JMA control run at a lead time of $72 \mathrm{~h}$. In addition, the NCEP control run had a small negative forecast error over the northern Rocky Mountains at a 72-h lead time, corresponding to a small trough; the JMA control run did not produce this error.

With increasing forecast time, the negative error (the trough) over the northern Rocky Mountains in the 

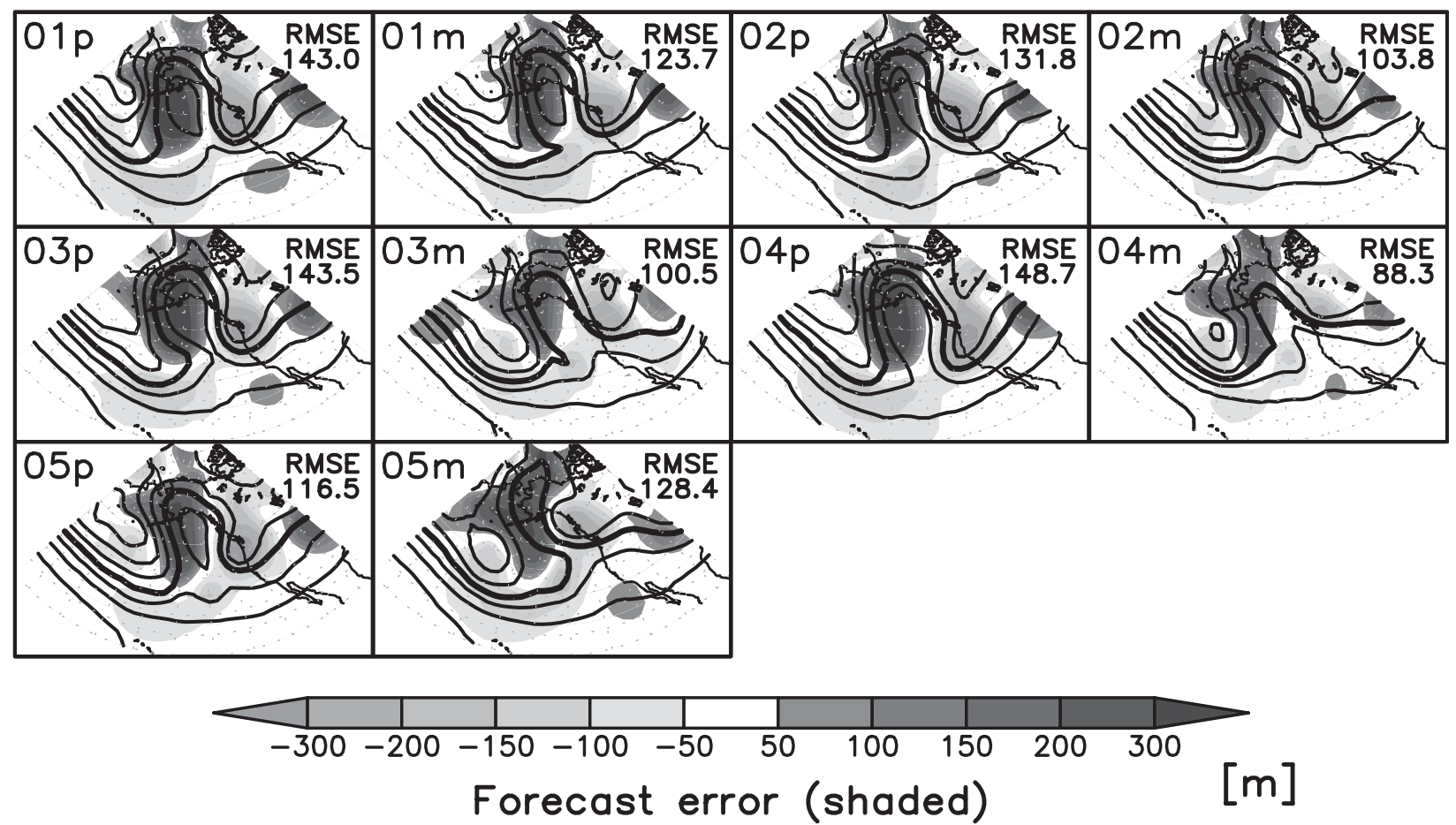

FIG. 4. NCEP EPS perturbed runs initialized at 1200 UTC 10 Dec 2005, showing the 120-h forecast of 500-hPa height (contours) and forecast error (shading). The letters "p" and " $m$ " used with the numbers in the upper-left corner indicate ensemble members for which the initial perturbation was added to and subtracted from the control run, respectively. The thick contours indicate 5500 gpm. RMSE is calculated over the blocking region $\left(20^{\circ}-80^{\circ} \mathrm{N}, 170^{\circ} \mathrm{E}-100^{\circ} \mathrm{W}\right)$.

NCEP control run showed a rapid development while traveling southward along the mountains. At 120-h lead time, the trough appears to block an eastward shift of the blocking ridge, leading to the generation of blocking that was different from that observed. The 120-h RMSE of the NCEP control run is about 1.7 times that of the JMA control run. The origin of the trough over the northern Rocky Mountains at 72-h lead time in the NCEP control run appears to be a small trough over Alaska at a lead time of $48 \mathrm{~h}$. Although the JMA control run also produced a small trough over Alaska at a lead time of $48 \mathrm{~h}$, the absence of a cutoff cyclone appears to explain the correct development of the blocking ridge at a lead time of $72 \mathrm{~h}$, which in turn meant that the small trough over Alaska at a lead time of $48 \mathrm{~h}$ remaining stationary until a lead time of $72 \mathrm{~h}$.

The blocking with remarkable meandering was also predicted by the NCEP perturbed runs (Fig. 4), possibly suggesting that the NCEP initial perturbation performed poorly in reducing uncertainties in the control analysis at the initial time of the ensemble forecast. For the area over the Rocky Mountains at a lead time of $120 \mathrm{~h}$, all the NCEP perturbed members had a trough (a negative forecast error) that blocked the eastward shift of the blocking ridge, as in the NCEP control run. The perturbed member $04 \mathrm{~m}$ ( $\mathrm{m}$ indicates an ensemble member for which the initial perturbation is subtracted from the control run) showed better performance in forecasting blocking formation than did the other members, but worse performance than the JMA control run.

\section{c. JMA GSM multianalysis ensemble forecasts with NCEP EPS analyses}

To determine the causes of the NCEP collective misprediction, multianalysis ensemble forecasts were conducted using the JMA GSM (TL159L40) and NCEP EPS control and perturbed analyses.

Figure $3 \mathrm{~d}$ shows the time evolution of the JMA GSM run from the NCEP control analysis. Up to a lead time of $48 \mathrm{~h}$, there were no apparent differences between the NCEP control run and the JMA GSM run. At a lead time of $72 \mathrm{~h}$, however, a cutoff cyclone over the North Pacific in the JMA GSM run was weaker than that in the NCEP control run, resulting in a smaller forecast error over the blocking region. At a lead time of $120 \mathrm{~h}$, the JMA GSM run incorrectly predicted the location of the blocking ridge, as in the NCEP control run, although the forecast error over the blocking region was reduced. Given that replacement of the NCEP operational model with the JMA GSM appears to have no influence on the 


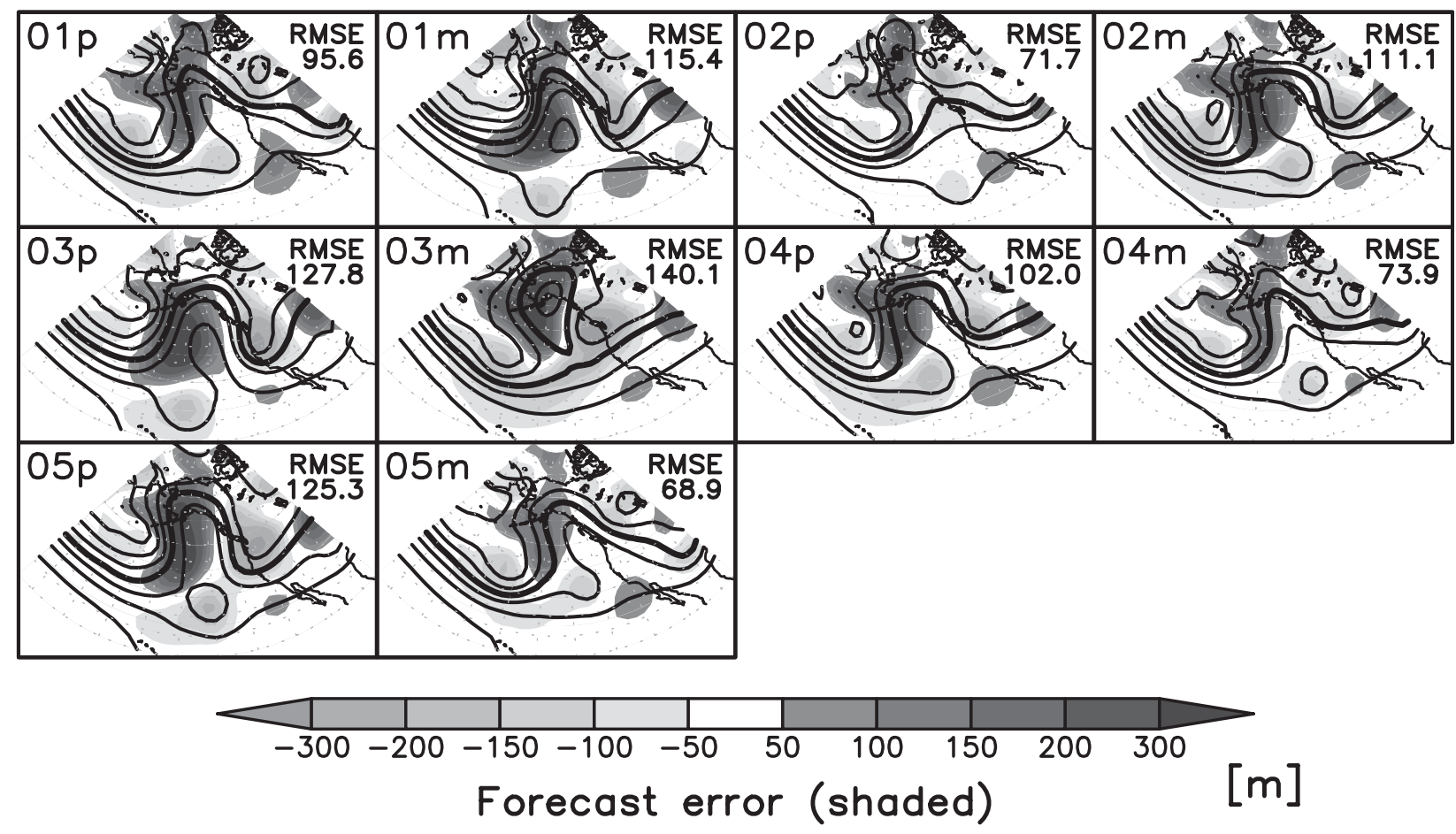

FIG. 5. As in Fig. 4, but for the JMA GSM runs from the NCEP EPS perturbed analyses.

predicted location of the blocking ridge, it might be concluded that one cause of the NCEP collective misprediction of blocking location was the NCEP control analysis.

The JMA GSM runs from the NCEP perturbed analyses were also incorrect in terms of predicting the location of blocking (Fig. 5), as in the corresponding NCEP perturbed members. However, most of the JMA GSM runs from the NCEP perturbed analyses were more accurate in predicted blocking location than were the corresponding NCEP perturbed members. As shown in the third column from the left in Table 2, the JMA GSM runs from the NCEP analyses, except for $02 \mathrm{~m}, 03 \mathrm{~m}$, and $05 \mathrm{p}$ ( $\mathrm{p}$ indicates an ensemble member for which the initial perturbation is added to the control run), had a smaller forecast error over the blocking region than did the corresponding NCEP perturbed members. In particular, members $02 \mathrm{p}, 04 \mathrm{~m}$, and $05 \mathrm{~m}$ had especially small forecast errors over the blocking region; those errors are smaller than that in the JMA EPS control run. A similar result was obtained for forecast skill over the NH (third column from the left in Table 3).

TABLE 2. 120-h RMSEs (in gpm) of NCEP EPS members and JMA GSM runs with NCEP EPS, globally amplified NCEP, and regionally amplified NCEP analyses for 500 -hPa height over the blocking region $\left(20^{\circ}-80^{\circ} \mathrm{N}, 170^{\circ} \mathrm{E}-100^{\circ} \mathrm{W}\right)$.

\begin{tabular}{|c|c|c|c|c|}
\hline \multirow[b]{2}{*}{1200 UTC 10 Dec $2005+120 \mathrm{~h}$} & \multirow[b]{2}{*}{ NCEP EPS members } & \multicolumn{3}{|c|}{ JMA GSM runs with NCEP analyses } \\
\hline & & Amplified: 1.0 & Amplified: 1.5 & Amplified: 1.5 area \\
\hline 00 & 139.2 & 122.7 & - & - \\
\hline $01 \mathrm{p}$ & 143.0 & 95.6 & 84.1 & 81.9 \\
\hline $01 \mathrm{~m}$ & 123.7 & 115.4 & 136.8 & 128.9 \\
\hline $02 p$ & 131.8 & 71.7 & 71.3 & 61.9 \\
\hline $02 \mathrm{~m}$ & 103.8 & 111.1 & 101.0 & 110.0 \\
\hline $03 p$ & 143.5 & 127.8 & 141.8 & 134.5 \\
\hline $03 \mathrm{~m}$ & 100.5 & 140.1 & 151.9 & 138.6 \\
\hline $04 p$ & 148.7 & 102.0 & 89.5 & 93.7 \\
\hline $04 \mathrm{~m}$ & 88.3 & 73.9 & 68.0 & 56.5 \\
\hline $05 p$ & 116.5 & 125.3 & 98.4 & 101.3 \\
\hline $05 \mathrm{~m}$ & 128.4 & 68.9 & 63.7 & 63.1 \\
\hline Ensemble mean & 117.1 & 91.1 & 78.0 & 79.6 \\
\hline
\end{tabular}


TABLE 3. As in Table 2, but for the Northern Hemisphere $\left(20^{\circ}-90^{\circ} \mathrm{N}\right)$.

\begin{tabular}{|c|c|c|c|c|}
\hline \multirow[b]{2}{*}{1200 UTC 10 Dec $2005+120 \mathrm{~h}$} & \multirow[b]{2}{*}{ NCEP EPS members } & \multicolumn{3}{|c|}{ JMA GSM runs with NCEP analyses } \\
\hline & & Amplified: 1.0 & Amplified: 1.5 & Amplified: 1.5 area \\
\hline 00 & 96.9 & 87.1 & - & - \\
\hline $01 \mathrm{p}$ & 104.6 & 79.0 & 82.6 & 75.8 \\
\hline $01 \mathrm{~m}$ & 90.8 & 104.1 & 122.1 & 109.6 \\
\hline $02 \mathrm{p}$ & 102.6 & 69.0 & 88.3 & 66.5 \\
\hline $02 \mathrm{~m}$ & 82.7 & 97.0 & 104.2 & 97.4 \\
\hline $03 p$ & 110.1 & 105.2 & 117.0 & 108.8 \\
\hline $03 \mathrm{~m}$ & 79.1 & 87.5 & 95.5 & 86.8 \\
\hline $04 \mathrm{p}$ & 99.3 & 79.9 & 78.3 & 78.6 \\
\hline $04 \mathrm{~m}$ & 81.2 & 67.0 & 72.9 & 63.1 \\
\hline $05 p$ & 96.3 & 87.6 & 76.4 & 80.2 \\
\hline $05 \mathrm{~m}$ & 98.4 & 78.4 & 85.7 & 76.2 \\
\hline Ensemble mean & 85.8 & 72.5 & 70.7 & 69.1 \\
\hline
\end{tabular}

The ensemble mean forecast of the JMA GSM runs from NCEP analyses also shows reductions in forecast errors over both the blocking region and the $\mathrm{NH}$.

For most members, forecasts were improved by replacing the NCEP operational model with the JMA GSM. This reduction in forecast errors with the introduction of a different numerical model was achieved via a reduction in imperfections of the model formulation, representing an advantage of the multimodel ensemble approach in predicting high-impact weather.

\section{d. Ensemble-based sensitivity analysis}

To identify the sensitive area for the blocking formation, an ensemble-based sensitivity analysis (as proposed by Enomoto et al. 2007) was performed using the
JMA ensemble forecast data initialized at 1200 UTC 10 December 2005. The dry total energy norm (Ehrendorfer and Errico 1995; Talagrand 1981) was used, as follows:

$$
\mathrm{TE}=\frac{1}{2} \iint_{A} u^{\prime 2}+v^{\prime 2}+\frac{c_{p}}{T_{r}} T^{\prime 2}+R T_{r}\left(\frac{p_{s}^{\prime}}{p_{r}}\right)^{2} d A d p,
$$

where $u^{\prime}, v^{\prime}, T^{\prime}$, and $p_{s}^{\prime}$ are perturbed components of zonal and meridional velocity, temperature, and surface pressure, respectively; $c_{p}$ is the specific heat at constant pressure; $R$ is the gas constant for dry air; and $T_{r}(=300 \mathrm{~K})$ and $p_{r}(=800 \mathrm{hPa})$ are the reference temperature and

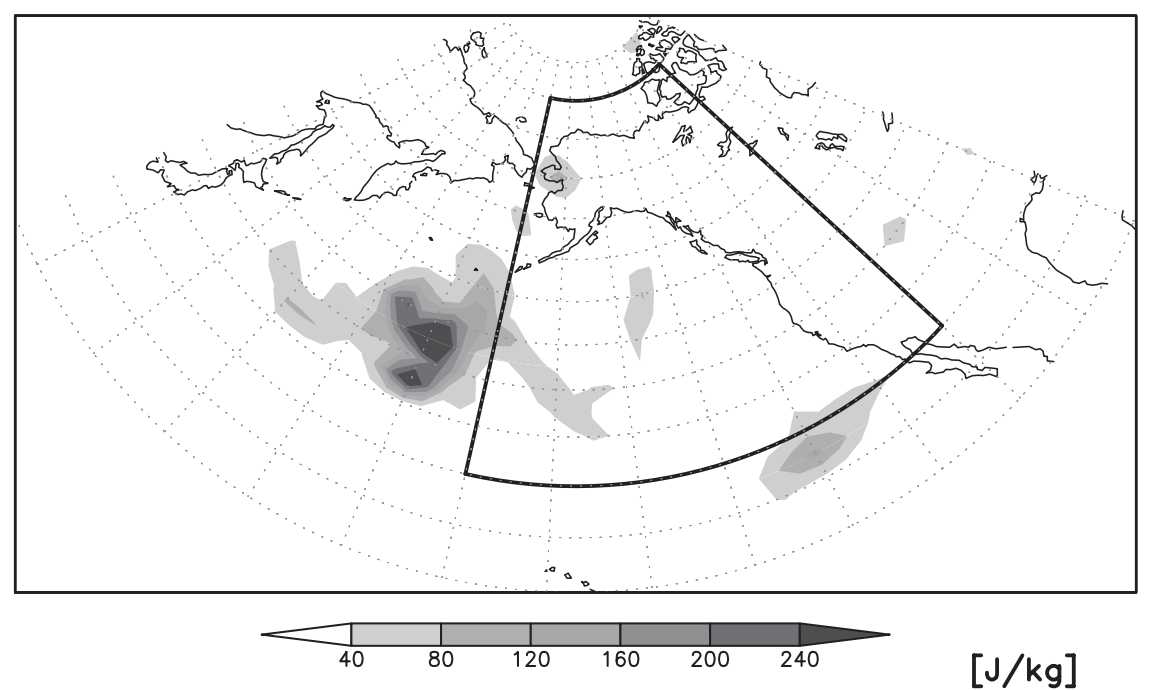

FIG. 6. Sensitive area for blocking formation, as detected by an ensemble-based sensitivity analysis with the JMA ensemble forecast initialized at 1200 UTC 10 Dec 2005, measured by dry total energy. The target time is 1200 UTC $15 \mathrm{Dec} 2005$. The target area is bounded by the solid black line $\left(30^{\circ}-75^{\circ} \mathrm{N}, 170^{\circ}-110^{\circ} \mathrm{W}\right)$. 


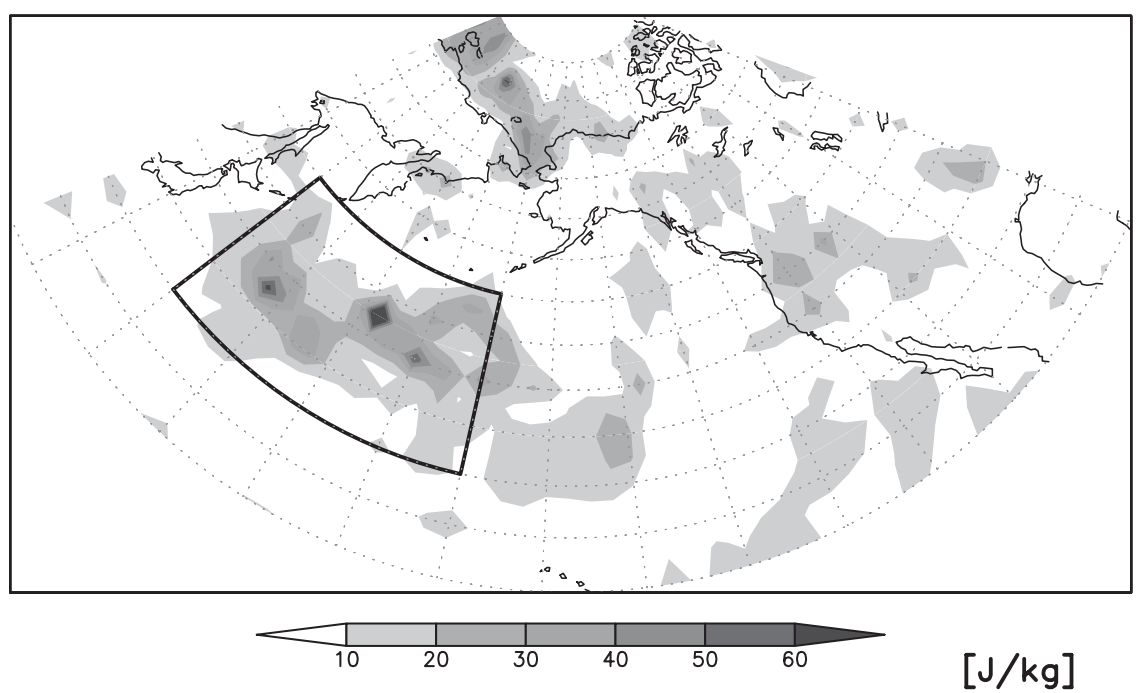

FIG. 7. Initial difference between the JMA EPS and NCEP EPS control analyses at 1200 UTC 10 Dec 2005, measured by dry total energy. A sensitive area for blocking formation is bounded by the solid black line $\left(30^{\circ}-50^{\circ} \mathrm{N}, 150^{\circ} \mathrm{E}-170^{\circ} \mathrm{W}\right)$.

pressure, respectively. A target area is set at $30^{\circ}-75^{\circ} \mathrm{N}$, $170^{\circ}-110^{\circ} \mathrm{W}$ and $1000-200 \mathrm{hPa}$ (solid line in Fig. 6). The target time is 1200 UTC 15 December 2005 (i.e., a lead time of $120 \mathrm{~h}$ ).

Figure 6 shows a sensitive area obtained from JMA ensemble data, measured by the vertically integrated dry total energy norm for the fastest-growing initial perturbation $\mathbf{x}$ in Eq. (3). A well-defined signal was detected over the central North Pacific, for components $u, v$, and $T$, at each pressure level (data not shown). This region was defined as a sensitive area $\left(30^{\circ}-50^{\circ} \mathrm{N}, 150^{\circ} \mathrm{E}-\right.$ $170^{\circ} \mathrm{W}$; area bounded by the solid line in Fig. 7). In this area, the analysis differences between the JMA and NCEP control runs at 1200 UTC 10 December 2005 (as measured by the dry total energy norm, for which perturbed components were replaced with analysis differences between the control runs) were larger than those in other areas (Fig. 7). This finding may suggest that the analysis had large uncertainties in the sensitive area. The differences appear to have arisen because of the analysis of a cutoff cyclone in the sensitive area (Fig. 8), as also seen in the Z500 field. The pressure difference between the JMA and NCEP control analyses at the center of the cutoff cyclone $\left(40^{\circ} \mathrm{N}, 180^{\circ}\right)$ was about $4 \mathrm{hPa}$ at $1200 \mathrm{UTC}$ 10 December 2005 , increasing to $\sim 8 \mathrm{hPa}$ at a lead time of $48 \mathrm{~h}$. For lead times greater than $72 \mathrm{~h}$, the predicted cutoff cyclones traveled in somewhat different directions: the cutoff cyclone in the NCEP control run traveled toward the southeast (as seen in the Z500 field; Fig. 3), whereas that in the JMA control run traveled toward the northeast. Given the above predictions, the synoptic field around the cutoff cyclone over the central North Pacific at 1200 UTC 10 December might be considered to have affected the development of blocking.

\section{e. JMA GSM multianalysis ensemble forecasts with amplified NCEP analyses}

Even in the case that a control analysis has large initial uncertainties, it is possible that the uncertainties would be reduced by the initial perturbations in ensemble forecasts. Figure 9 shows the dry total energy for the NCEP initial perturbations at 1200 UTC 10 December 2005. The initial perturbations, 02 and 03 , did not have well-defined signals around the cutoff cyclone over the central North Pacific. Even in the other perturbations (01, 04, and 05), which had signals around the cutoff cyclone, the amplitude of initial perturbations was smaller than the analysis difference shown in Fig. 7. These results may suggest that the NCEP initial perturbations did not operate effectively in improving the incorrect blocking prediction by the NCEP control run. It is possible that amplification of the NCEP initial perturbations results in improved blocking prediction.

First, JMA GSM multianalysis ensemble forecasts were conducted with the globally amplified NCEP perturbed analyses. The amplitude of the NCEP initial perturbations was increased by a factor of 1.5. Comparisons between the third and fourth columns from the left in Table 2 reveal the influence of global amplification of the NCEP initial perturbations on the 120-h RMSE for Z500 over the blocking region $\left(20^{\circ}-80^{\circ} \mathrm{N}\right.$, $\left.170^{\circ} \mathrm{E}-100^{\circ} \mathrm{W}\right)$. Most of the runs, including the ensemblemean forecast, produced reduced forecast errors over the blocking region due to global amplification of the initial 


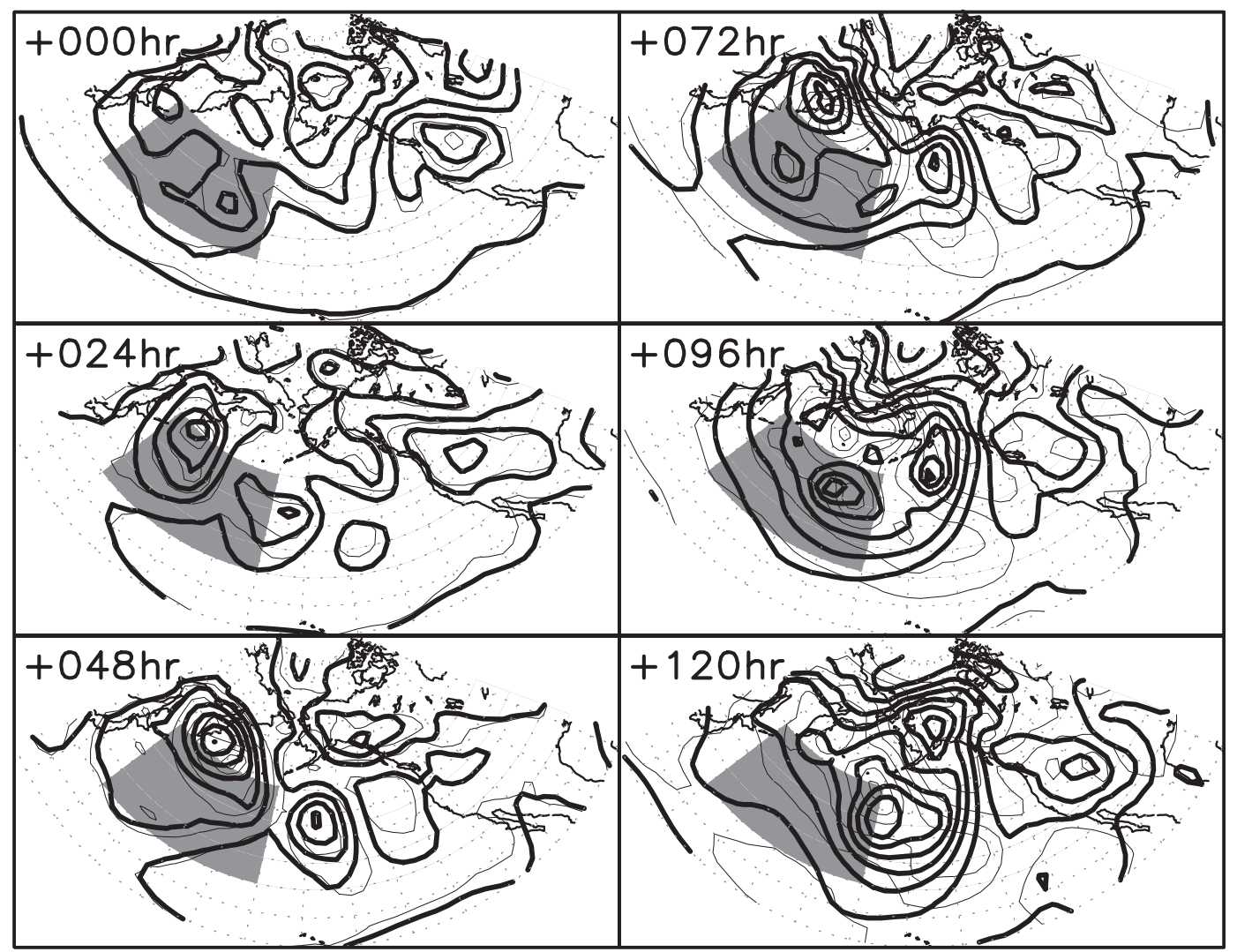

FIG. 8. Time evolution of sea level pressure for the JMA EPS (thick contours) and the NCEP EPS (thin contours) control runs initialized at $1200 \mathrm{UTC} 10 \mathrm{Dec} 2005$. Shading indicates a sensitive area $\left(30^{\circ}-50^{\circ} \mathrm{N}, 150^{\circ} \mathrm{E}-170^{\circ} \mathrm{W}\right)$ for blocking formation, as detected by the ensemble-based sensitivity analysis.

perturbations, although this also resulted in increased forecast errors over the $\mathrm{NH}$, except for $04 \mathrm{p}, 05 \mathrm{p}$, and the ensemble mean (third and fourth columns from the left in Table 3). This finding may suggest that excessive amplification of initial perturbations over nonsensitive areas is undesirable.

Next, additional JMA GSM multianalysis ensemble forecasts were performed with regionally amplified NCEP perturbed analyses. The amplitude of the NCEP initial perturbations was increased by a factor of 1.5 , although only over the sensitive area detected by the ensemble-based sensitivity analysis. For most members, the regional amplification resulted in reduced forecast error over the blocking region without any degradation of forecasts over the $\mathrm{NH}$ (third and fifth columns from the left in Tables 2 and 3).

It can be concluded that the sensitive area detected by the ensemble-based sensitivity analysis plays a crucial role in blocking formation. A part of the regionally amplified members, 01p, 02p, 04m, and 05m, have lower RMSE over the blocking region than do the other corresponding members. The location of blocking predicted by these members (Fig. 10) is closer to the observed location than that predicted by the corresponding NCEP members (Fig. 4). These members did not have welldefined negative forecast errors (troughs) over the Rocky Mountains at 120-h lead time. The absence of troughs appears to have enabled the blocking ridge to shift eastward.

In terms of ensemble mean forecast, that obtained from the regionally amplified members has a smaller forecast error over the $\mathrm{NH}$ than does that obtained from the globally amplified members (bottom line in Table 3 ), whereas the former has a larger forecast error over the blocking region than the latter (bottom line in Table 2). However, it would be most appropriate to evaluate forecast performance in terms of high-impact weather by using individual ensemble members rather than an ensemble mean forecast, as the latter cannot provide the occurrence probabilities of high-impact weather. Although the improvement in forecasts due to regional amplification of initial perturbations was not observed for all members, it is clear that the probabilistic forecasts of blocking were improved. 


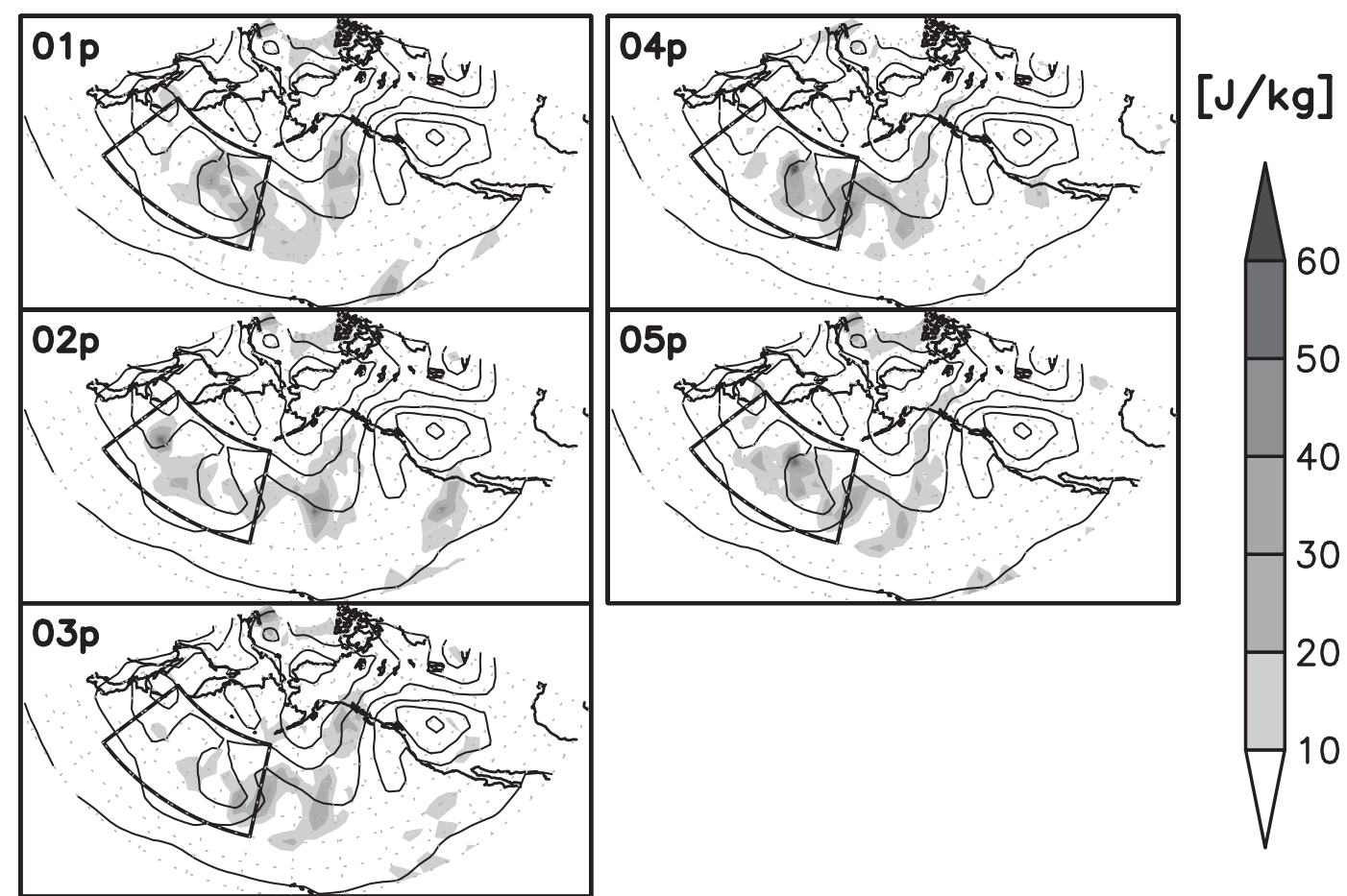

FIG. 9. Sea level pressure for the NCEP EPS control run (thin contours) and dry total energy for NCEP EPS initial perturbations (shading) at $1200 \mathrm{UTC} 10 \mathrm{Dec} 2005$. A sensitive area is bounded by the thick black line $\left(30^{\circ}-50^{\circ} \mathrm{N}\right.$, $\left.150^{\circ} \mathrm{E}-170^{\circ} \mathrm{W}\right)$.

\section{Conclusions}

An atmospheric blocking event occurred over the Rocky Mountains at 1200 UTC 15 December 2005. The operational medium-range ensemble forecasts of CMC, JMA, and NCEP, as initialized at 1200 UTC 10 December 2005, showed remarkable differences regarding this event. All the NCEP members failed to predict the correct location of the blocking, whereas almost all the JMA members and most of the CMC members were successful in this regard. The causes of the NCEP collective misprediction were investigated using an ensemble-based sensitivity analysis and JMA GSM multianalysis ensemble forecasts with NCEP, regionally amplified NCEP, and globally amplified NCEP analyses.

Although blocking prediction was improved by replacing the numerical model, the NCEP collective misprediction resulted from problems in the NCEP control analysis over the central North Pacific at the initial time of the ensemble forecast. The initial differences between the JMA and NCEP control runs were larger over the central North Pacific (related to a cutoff cyclone) than over other areas. This finding suggests the existence of large uncertainties around the cutoff cyclone. Accurate prediction of the cutoff cyclone was essential for the correct prediction of blocking formation. Another cause of the collective misprediction is that the NCEP initial perturbations did not operate effectively in reducing uncertainties in the NCEP control analysis.

Although it is beyond the scope of this paper to identify the causes of initial differences between the JMA and NCEP control runs, possible factors are differences in the data assimilation system, the observation, and the satellite data used in each data assimilation system. In recent years, many Observing System Experiments (OSEs) have been conducted throughout the world to investigate the impacts of observations on analysis and forecast errors in downstream areas (e.g., Langland et al. 1999; Szunyogh et al. 2002; Langland 2005; Fourrie et al. 2006; Wu et al. 2007; Chou and Wu 2008; Sellwood et al. 2008; Inoue et al. 2009; Yamaguchi et al. 2009). In addition, TIGGE has enabled us to obtain operational medium-range ensemble forecast data quasi-operationally ( $\sim 2$ day behind). OSEs and TIGGE can be used to investigate quasioperationally the factors that result in interanalysis and intermodel differences in predictions of high-impact weather such as blocking, thereby leading to more valuable and useful ensemble forecasts under collaborations between NWP centers, universities, and research institutes.

For most of the members in the present study, replacement of the NCEP operational model with the JMA GSM led to a reduction in forecast error over both 

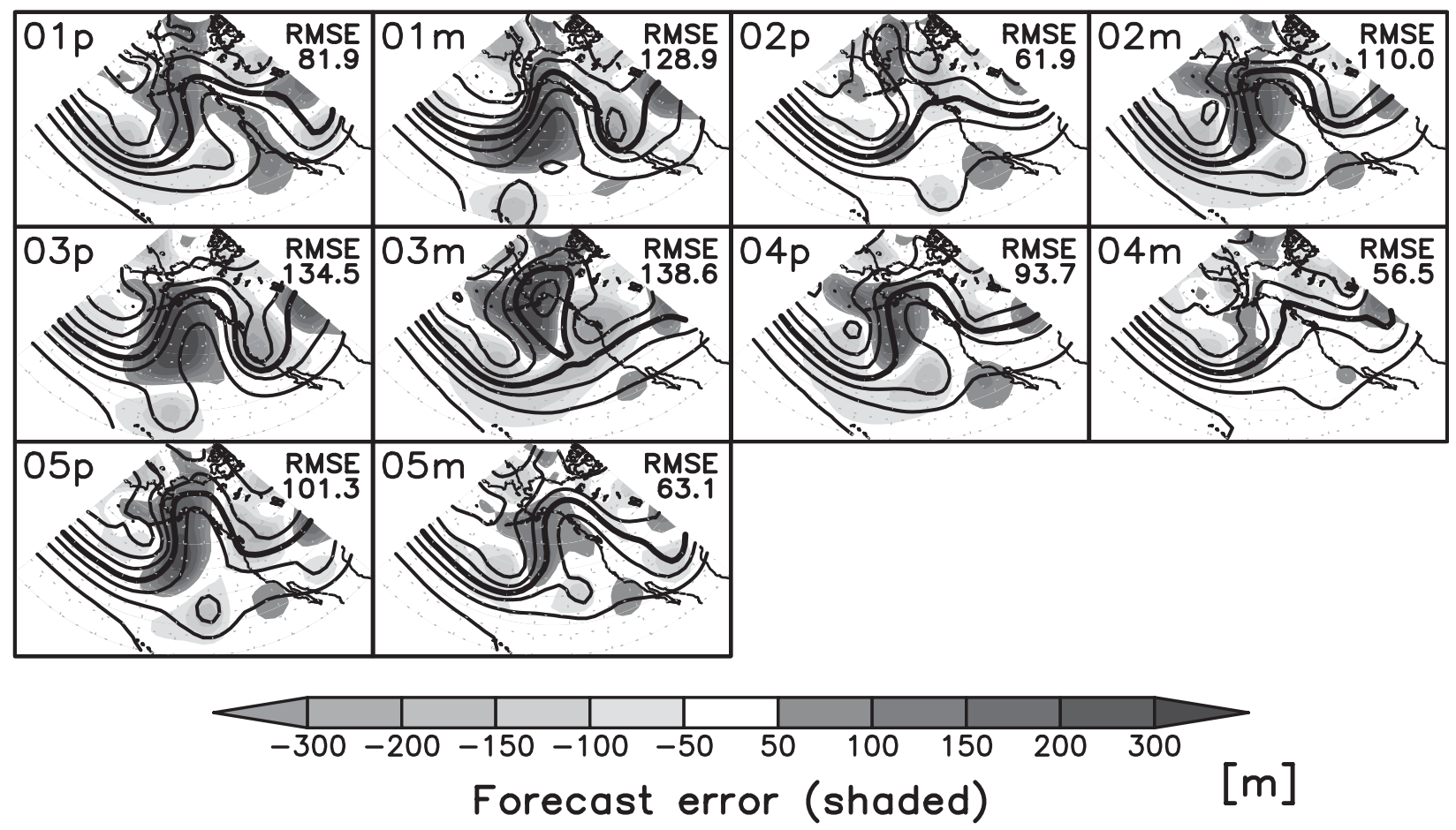

FIG. 10. As in Fig. 4, but for the JMA GSM runs from the regionally amplified (by a factor of 1.5) NCEP perturbed analyses, although only over the sensitive area.

the blocking region and the $\mathrm{NH}$, thereby demonstrating the advantage of the multimodel ensemble approach in predicting high-impact weather. The JMA GSM multianalysis ensemble forecasts revealed that regional amplification of initial perturbations over a sensitive area can lead to further improvements in forecasts over the blocking region, without any degradation of forecasts over the $\mathrm{NH}$, whereas global amplification of initial perturbations could lead to both improved forecasts over the blocking region and degraded forecasts over the NH. This finding may suggest that the excessive amplification of initial perturbations over nonsensitive areas is undesirable, and that a case-dependent rescaling of initial perturbations may have real value compared with climatology-based rescaling, which is used widely in current operational EPSs. There are large differences in RMSE between ensemble members with positive perturbations and ensemble members with negative perturbations in the NCEP EPS and the JMA GSM runs with NCEP and amplified NCEP analyses, over both the blocking region and the $\mathrm{NH}$. This suggests that using positive and negative pairs of initial perturbations (which are used widely in current operational EPSs) might be undesirable, at least for predictions of high-impact weather.

This study provides a good example of the fact that the performance of a particular ensemble forecast for high-impact weather can be improved by multimodel multianalysis (MMMA) ensemble approach with other ensemble forecast data and a numerical model, thereby demonstrating the likely feasibility of GIFS, whose objective is production of internationally coordinated advance warnings and forecasts for high-impact weather. However, there are not enough examples that demonstrate the advantage of MMMA ensemble approach in predicting high-impact weather. Further experiments of MMMA ensemble approach are needed with a focus on high-impact weather. Although there may be many difficulties in conducting MMMA ensemble forecast operationally (e.g., limitations of computer resources and transfer failure of initial data for model integrations), MMMA ensemble approach might help us not only to get reliable information on high-impact weather in advance, but also to know the factors that result in forecast differences among other NWP centers and to improve each ensemble forecast system.

Acknowledgments. The authors thank Dr. Richard Wobus and Dr. Mozheng Wei for providing ensemble forecast data with which to conduct model integrations, and for their valuable comments and discussions. The authors are grateful to the two anonymous reviewers for insightful suggestions. Part of this work was performed as part of collaboration between the Numerical 
Prediction Division of JMA and the University of Tsukuba.

\section{REFERENCES}

Black, E., M. Blackburn, G. Harrison, B. Hoskins, and J. Methven, 2004: Factors contributing to the summer 2003 European heatwave. Weather, 59, 217-223.

Carrera, M. L., R. W. Higgins, and V. E. Kousky, 2004: Downstream weather impacts associated with atmospheric blocking over the Northeast Pacific. J. Climate, 17, 4823-4839.

Cash, B. A., and S. Lee, 2000: Dynamical processes of block evolution. J. Atmos. Sci., 57, 3202-3218.

Chou, K. H., and C. C. Wu, 2008: Typhoon initialization in a mesoscale model-Combination of the bogused vortex and the dropwindsonde data in DOTSTAR. Mon. Wea. Rev., 136, 865-879.

Côté, J., S. Gravel, A. Méthot, A. Patoine, M. Roch, and A. Staniforth, 1998a: The operational CMC-MRB Global Environmental Multiscale (GEM) model. Part I: Design considerations and formulation. Mon. Wea. Rev., 126, 1373-1395.

_ J.-G. Desmarais, S. Gravel, A. Méthot, A. Patoine, M. Roch, and A. Staniforth, 1998b: The operational CMC-MRB Global Environmental Multiscale (GEM) model. Part II: Results. Mon. Wea. Rev., 126, 1397-1418.

D'Andrea, F., and Coauthors, 1998: Northern Hemisphere atmospheric blocking as simulated by 15 atmospheric general circulation models in the period 1979-1988. Climate Dyn., 14,385-407.

Dole, R. M., 1986: Persistent anomalies of the extratropical Northern Hemisphere wintertime circulation: Structure. Mon. Wea. Rev., 114, 178-207.

Ehrendorfer, M., and R. M. Errico, 1995: Mesoscale predictability and the spectrum of optimal perturbation. J. Atmos. Sci., 52, 3475-3500.

Enomoto, T., W. Ohfuchi, H. Nakamura, and M. A. Shapiro, 2007: Remote effects of tropical storm Cristobal upon a cut-off cyclone over Europe in August 2002. Meteor. Atmos. Phys., 96, $29-42$.

Fourrie, N., D. Marchal, F. Rabier, B. Chapnik, and G. Desroziers, 2006: Impact study of the 2003 North Atlantic THORPEX Regional Campaign. Quart. J. Roy. Meteor. Soc., 132, 275-295.

Froude, L. S. R., 2010: TIGGE: Comparison of the prediction of Northern Hemisphere extratropical cyclones by different ensemble prediction systems. Wea. Forecasting, 25, 819-836.

Houtekamer, P. L., and H. L. Mitchell, 2005: Ensemble Kalman filtering. Quart. J. Roy. Meteor. Soc., 131, 3269-3289.

Inoue, J., T. Enomoto, T. Miyoshi, and S. Yamane, 2009: Impact of observations from Arctic drifting buoys on the reanalysis of surface fields. Geophys. Res. Lett., 36, L08501, doi:10.1029/ 2009GL037380.

JMA, 2007: Outline of the operational numerical weather prediction at the Japan Meteorological Agency. Japan Meteorological Agency, $196 \mathrm{pp}$.

Johnson, C., and R. Swinbank, 2009: Medium-range multi-model ensemble combination and calibration. Quart. J. Roy. Meteor. Soc., 135, 777-794.

Kalnay, E., 2003: Atmospheric Modeling, Data Assimilation and Predictability. Cambridge University Press, $341 \mathrm{pp}$.

Kimoto, M., H. Mukougawa, and S. Yoden, 1992: Medium-range forecast skill variation and blocking transition: A case study. Mon. Wea. Rev., 120, 1616-1627.

Langland, R. H., 2005: Observation impact during the North Atlantic TReC-2003. Mon. Wea. Rev., 133, 2297-2309.
_ and Coauthors, 1999: The North Pacific Experiment (NORPEX98): Targeted observations for improved North American weather forecasts. Bull. Amer. Meteor. Soc., 80, 1363-1384.

Lejenäs, H., and H. Økland, 1983: Characteristics of Northern Hemisphere blocking as determined from a long time series of observational data. Tellus, 35A, 350-362.

Majumdar, S. J., and P. M. Finocchio, 2010: On the ability of global Ensemble Prediction Systems to predict tropical cyclone track probabilities. Wea. Forecasting, 25, 659-680.

Matsueda, M., 2009: Blocking predictability in operational mediumrange ensemble forecasts. SOLA, 5, 113-116.

— 2011: Predictability of Euro-Russian blocking in summer of 2010. Geophys. Res. Lett., 38, L06801, doi:10.1029/ 2010 GL046557.

, and H. L. Tanaka, 2008: Can MCGE outperform the ECMWF ensemble? SOLA, 4, 77-80.

—, M. Kyouda, H. L. Tanaka, and T. Tsuyuki, 2006: Multi-center grand ensemble using three operational ensemble forecasts. SOLA, 2, 33-36.

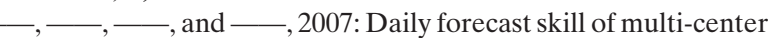
grand ensemble. SOLA, 3, 29-32.

_ - R. Mizuta, and S. Kusunoki, 2009: Future change in wintertime atmospheric blocking simulated using a $20-\mathrm{km}$-mesh atmospheric global circulation model. J. Geophys. Res., 114, D12114, doi:10.1029/2009JD011919.

— , H. Endo, and R. Mizuta, 2010: Future change in Southern Hemisphere summertime and wintertime atmospheric blockings simulated using a 20-km-mesh AGCM. Geophys. Res. Lett., 37, L02803, doi:10.1029/2009GL041758.

Mauritsen, T., and E. Källén, 2004: Blocking prediction in an ensemble forecasting system. Tellus, 56A, 218-228.

Nakamura, H., M. Nakamura, and J. L. Anderson, 1997: The role of high- and low-frequency dynamics in blocking formation. Mon. Wea. Rev., 125, 2074-2093.

Namias, J., 1947: Characteristics of the general circulation over the Northern Hemisphere during the abnormal winter 1946-47. Mon. Wea. Rev., 75, 145-152.

Palmer, T. N., F. J. Doblas-Reyes, A. Weisheimer, and M. J. Rodwell, 2008: Toward seamless prediction: Calibration of climate change projections using seasonal forecasts. Bull. Amer. Meteor. Soc., 89, 459-470.

Pappenberger, F., J. Bartholmes, J. Thielen, H. L. Cloke, R. Buizza, and A. de Roo, 2008: New dimensions in early flood warning across the globe using grand-ensemble weather predictions. Geophys. Res. Lett., 35, L10404, doi:10.1029/2008GL033837.

Park, Y.-Y., R. Buizza, and M. Leutbecher, 2008: TIGGE: Preliminary results on comparing and combining ensembles. Quart. J. Roy. Meteor. Soc., 134, 2029-2050.

Pelly, J. L., and B. J. Hoskins, 2003a: A new perspective on blocking. J. Atmos. Sci., 60, 743-755.

, and 2003b: How well does the ECMWF ensemble prediction system predict blocking? Quart. J. Roy. Meteor. Soc., 129, 1683-1702.

Quadrelli, R., V. Pavan, and F. Molteni, 2001: Wintertime variability of Mediterranean precipitation and its links with large-scale circulation anomalies. Climate Dyn., 17, 457466.

Rex, D. F., 1950: Blocking action in the middle troposphere and its effect upon regional climate I: An aerological study of blocking action. Tellus, 2, 196-211.

Richardson, D., R. Buizza, and R. Hagedorn, 2005: Final report of the First Workshop on the THORPEX Interactive Grand Global Ensemble (TIGGE). WMO TD 1273, WWRP-THORPEX 5, 39 pp. 
Ritchie, H., 1991: Application of the semi-Lagrangian method to a multilevel spectral primitive-equations model. Quart. J. Roy. Meteor. Soc., 117, 91-106.

, and C. Beaudoin, 1994: Approximations and sensitivity experiments with a baroclinic semi-Lagrangian spectral model. Mon. Wea. Rev., 122, 2391-2399.

Sellwood, J. K., J. S. Majumdar, E. B. Mapes, and I. Szunyogh, 2008: Predicting the influence of observations on medium-range forecasts of atmospheric flow. Quart. J. Roy. Meteor. Soc., 134, 2011-2027.

Shutts, G. J., 1983: The propagation of eddies in diffluent jet streams: Eddy vorticity forcing of "blocking" flow fields. Quart. J. Roy. Meteor. Soc., 109, 737-762.

- 1986: A case study of eddy forcing during an Atlantic blocking episode. Advances in Geophysics, Vol. 29, Academic Press, 135-162.

Szunyogh, I., Z. Toth, A. V. Zimin, S. J. Majumdar, and A. Persson, 2002: Propagation of the effect of targeted observations: The 2000 Winter Storm Reconnaissance program. Mon. Wea. Rev., 130, 1144-1165.

Talagrand, O., 1981: A study of the dynamics of four-dimensional data assimilation. Tellus, 33, 43-60.

Tanaka, H. L., and M. F. Milkovich, 1990: A heat budget analysis of the polar troposphere in and around Alaska during the abnormal winter of 1988/89. Mon. Wea. Rev., 118, 16281639.

Tibaldi, S., and F. Molteni, 1990: On the operational predictability of blocking. Tellus, 42A, 343-365.

Toth, Z., and E. Kalnay, 1993: Ensemble forecasting at NMC: The generation of perturbations. Bull. Amer. Meteor. Soc., 74, 2317-2330.

— , and — 1997: Ensemble forecasting at NCEP and the breeding method. Mon. Wea. Rev., 125, 3297-3319.

Trigo, R. M., I. F. Trigo, C. C. DaCamara, and T. J. Osborn, 2004: Climate impact of the European winter blocking episodes from the NCEP/NCAR reanalyses. Climate Dyn., 23, 17-28.

Tyrlis, E., and B. J. Hoskins, 2008: Aspects of a Northern Hemisphere atmospheric blocking climatology. J. Atmos. Sci., 65, 1638-1652.

WMO, 2005: THORPEX. WMO-978, 15 pp. , 2006: WMO at a glance. WMO-990, 20 pp.

Wu, C. C., K. H. Chou, P. H. Lin, S. D. Aberson, M. S. Peng, and T. Nakazawa, 2007: The impact of dropwindsonde data on typhoon track forecasts in DOTSTAR. Wea. Forecasting, 22, 1157-1176.

Yamaguchi, M., T. Iriguchi, T. Nakazawa, and C. C. Wu, 2009: An observing system experiment for Typhoon Conson (2004) using a singular vector method and DOTSTAR data. Mon. Wea. Rev., 137, 2801-2816. 